\title{
Congruence testing for odd subgroups of the modular group
}

\author{
Thomas Hamilton and David Loeffler
}

\begin{abstract}
We give a computationally effective criterion for determining whether a finite-index subgroup of $\mathrm{SL}_{2}(\mathbf{Z})$ is a congruence subgroup, extending earlier work of $\mathrm{Hsu}$ for subgroups of $\mathrm{PSL}_{2}(\mathbf{Z})$.
\end{abstract}

Recall that a finite-index subgroup of $\mathrm{SL}_{2}(\mathbf{Z})$ is said to be a congruence subgroup if it is defined by congruence conditions on the entries of its elements; formally, a subgroup is congruence if it contains the subgroup $\Gamma(N)$ of matrices congruent to the identity modulo $N$, and the least such $N$ is its level.

We are interested in the following question.

Question. Is there an efficient procedure that will determine whether a finite-index subgroup of $\mathrm{SL}_{2}(\mathbf{Z})$ is congruence?

One such algorithm follows from the following theorem, proved in [3], which is an extension of a classical theorem of Wolfahrt.

Theorem 1 (Kiming-Schütt-Verrill). Let $\Gamma \leqslant \mathrm{SL}_{2}(\mathbf{Z})$ be a finite-index subgroup, and let $d$ be the lowest common multiple of the widths of the cusps of $\Gamma$. If $\Gamma$ is congruence, then its level is either $d$ or $2 d$.

(The case of level $2 d$ can only occur if $\Gamma$ is odd, that is does not contain -1 .)

In principle, one can now determine whether $\Gamma$ is congruence by calculating explicitly a list of generators for $\Gamma(N)$, where $N=d$ or $2 d$ as appropriate, and testing whether each of these is contained in $\Gamma$. This approach is used in $[\mathbf{3}]$ in order to give explicit examples of non-congruence lifts to $\mathrm{SL}_{2}(\mathbf{Z})$ of congruence subgroups of $\mathrm{PSL}_{2}(\mathbf{Z})$. However, the number of generators of $\Gamma(N)$ grows rather quickly with $N$, so this algorithm rapidly becomes impractical for large values of $N$.

We present the following alternative approach to the above problem. As has been noted by Hsu [2] and others, a convenient data structure for representing a subgroup of $\mathrm{SL}_{2}(\mathbf{Z})$ of index $m$ is by the homomorphism $\mathrm{SL}_{2}(\mathbf{Z}) \rightarrow S_{m}$ given by left multiplication on the cosets $\mathrm{SL}_{2}(\mathbf{Z}) / \Gamma$. This, in turn, can be represented by two permutations giving the action of the generators $L=\left(\begin{array}{ll}1 & 0 \\ 1 & 1\end{array}\right)$ and $R=\left(\begin{array}{ll}1 & 1 \\ 0 & 1\end{array}\right)$ of $\mathrm{SL}_{2}(\mathbf{Z})$ on the cosets $\mathrm{SL}_{2}(\mathbf{Z}) / \Gamma$.

The computer algebra package Sage contains a library of routines for working with subgroups defined in this way, implemented by Vincent Delecroix and the second author based on an earlier implementation by Chris Kurth.

Theorem 2. Let $N=d$ if $-1 \in \Gamma$ and $N=2 d$ otherwise. Then there exists an explicit list of relations $\mathcal{L}_{N}$ in $L$ and $R$ (of length $\leqslant 7$ ), such that $\Gamma$ is congruence if and only if the permutation representation of $\mathrm{SL}_{2}(\mathbf{Z})$ corresponding to $\Gamma$ satisfies the relations in $\mathcal{L}_{N}$.

Received 2 July 2013; revised 6 November 2013.

2010 Mathematics Subject Classification 20H05 (primary). 
This theorem has been proved for subgroups containing -1 by Hsu [2]; our proof follows Hsu's closely, except that we use the Kiming-Schütt-Verrill theorem (Theorem 1) in place of the classical theorem of Wolfahrt.

Proposition 3. Let $N \geqslant 1$. There is an explicit finite set $\mathcal{L}_{N}$ of words in $L$ and $R$ whose image in $\mathrm{SL}_{2}(\mathbf{Z})$ normally generates $\Gamma(N)$ (that is, $\Gamma(N)$ is the smallest normal subgroup of $\mathrm{SL}_{2}(\mathbf{Z})$ containing the elements in $\left.\mathcal{L}_{N}\right)$.

Proof. See [2, Theorem 2.4]. The starting-point of the proof is the well-known fact that $\mathrm{SL}_{2}(\mathbf{Z})$ has the presentation

$$
\left\langle L, R \mid\left(L R^{-1} L\right)^{2}\left(R^{-1} L\right)^{-3},\left(L R^{-1} L\right)^{4}\right\rangle
$$

where $L$ and $R$ correspond to the matrices given above. Thus if $\mathcal{L}$ is any set of words in $L$ and $R$, the group

$$
\left\langle L, R \mid\left(L R^{-1} L\right)^{2}\left(R^{-1} L\right)^{-3},\left(L R^{-1} L\right)^{4}, \mathcal{L}\right\rangle
$$

is the largest quotient of $\mathrm{SL}_{2}(\mathbf{Z})$ in which the elements in the image of $\mathcal{L}$ map to the identity, which is the quotient of $\mathrm{SL}_{2}(\mathbf{Z})$ by the subgroup normally generated by the image of $\mathcal{L}$. In particular, the images of the elements of $\mathcal{L}$ normally generate $\Gamma(N)$ if and only if $(1)$ is a presentation of the finite group $\mathrm{SL}_{2}(\mathbf{Z} / N \mathbf{Z})$.

Explicit presentations of the groups $\mathrm{SL}_{2}(\mathbf{Z} / N \mathbf{Z})$ for all $N$ in terms of the generators $L$ and $R$ are given in [2, Lemmas 3.3-3.5] (based on earlier work of Behr and Mennicke [1]), so it suffices to take $\mathcal{L}_{N}$ to be the set of relations appearing in these presentations.

Proof of Theorem 2. Let $N$ be as defined in the statement of the theorem. We know that $\Gamma$ is congruence if and only if it contains $\Gamma(N)$. Let $\Gamma^{\prime}$ be the normal core of $\Gamma$, that is the intersection of the conjugates of $\Gamma$ in $\mathrm{SL}_{2}(\mathbf{Z})$; then, since the elements of $\mathcal{L}_{N}$ normally generate $\Gamma(N)$, it follows that $\Gamma$ is congruence if and only if $\mathcal{L}_{N} \subset \Gamma^{\prime}$.

However, $\Gamma^{\prime}$ is precisely the kernel of the map $\phi: \mathrm{SL}_{2}(\mathbf{Z}) \rightarrow S_{m}$ giving the permutation representation of $\Gamma$. So $\Gamma$ is congruence if and only if $\phi$ is trivial on the elements of $\mathcal{L}_{N}$.

(One could clearly adapt this argument to work with other explicit descriptions of $\Gamma$ as long as one has an algorithm for computing whether a given element of $\mathrm{SL}_{2}(\mathbf{Z})$ lies in the normal core of $\Gamma$.)

We now reproduce, for the reader's convenience, an explicit list of relations $\mathcal{L}_{N}$ as in Theorem 2, based on those given by Hsu.

- If $N$ is odd, one may take $\mathcal{L}_{N}$ to contain the single relation

$$
\left(R^{2} L^{-1 / 2}\right)^{3}=1
$$

where $\frac{1}{2}$ is the multiplicative inverse of $2 \bmod N$. This follows from the fact that for $N$ odd,

$$
\left\langle L, R \mid L^{N}=1,\left(L R^{-1} L\right)^{2}=\left(R^{-1} L\right)^{3},\left(L R^{-1} L\right)^{4}=1,\left(R^{2} L^{-1 / 2}\right)^{3}=1\right\rangle
$$

is a presentation of $\mathrm{SL}_{2}(\mathbf{Z} / N \mathbf{Z})$, by [2, Lemma 3.3]. The relations $\left(L R^{-1} L\right)^{2}=\left(R^{-1} L\right)^{3}$ and $\left(L R^{-1} L\right)^{4}=1$ are redundant; they are automatically satisfied by the permutation representation of $\mathrm{SL}_{2}(\mathbf{Z})$ corresponding to $\Gamma$, since they are satisfied in $\mathrm{SL}_{2}(\mathbf{Z})$ itself. The relation $L^{N}=1$ is also automatically satisfied, since by definition $N$ is divisible by the widths of all of the cusps of $\Gamma$. (This case can, of course, only occur if $-1 \in \Gamma$ and is thus identical to the first case of Hsu's Theorem 3.1.) 
- If $N$ is a power of 2 , let $S=L^{20} R^{1 / 5} L^{-4} R^{-1}$, where $\frac{1}{5}$ is the multiplicative inverse of $5 \bmod N$. Then one may take $\mathcal{L}_{N}$ to consist of the three relations

$$
\begin{aligned}
\left(L R^{-1} L\right)^{-1} S\left(L R^{-1} L\right) & =S^{-1}, \\
S^{-1} R S & =R^{25}, \\
\left(S R^{5} L R^{-1} L\right)^{3} & =\left(L R^{-1} L\right)^{2} .
\end{aligned}
$$

As in the previous case, this follows from the fact that

$$
\left\langle L, R \mid L^{N}=1,\left(L R^{-1} L\right)^{2}=\left(R^{-1} L\right)^{3},\left(L R^{-1} L\right)^{4}=1, \mathcal{L}_{N}\right\rangle
$$

is a presentation of $\mathrm{SL}_{2}(\mathbf{Z} / N \mathbf{Z})$, by [2, Lemma 3.4], and the first three relations are automatically satisfied in the permutation relation corresponding to $\Gamma$.

(Note that if we assume that $-1 \in \Gamma$, we may replace the last relation with $\left(S R^{5} L R^{-1} L\right)^{3}=1$, which is the relation appearing in Hsu's Theorem 3.1; but for odd subgroups we must use the slightly more complicated relation above.)

- If $N=e m$ where $e$ is a power of $2, m$ is odd and $e, m>1$, then let $c, d$ be the unique integers $\bmod N$ such that $c=0 \bmod e, c=1 \bmod m, d=1 \bmod e, d=0 \bmod m$. Write $a=L^{c}, b=R^{c}, l=L^{d}, r=R^{d}$ and $s=l^{20} r^{1 / 5} l^{-4} r^{-1}$, where $\frac{1}{5}$ is interpreted $\bmod e$. Then we may take $\mathcal{L}_{N}$ to consist of the seven elements

$$
\begin{aligned}
{[a, r] } & =1, \\
\left(a b^{-1} a\right)^{4} & =1, \\
\left(a b^{-1} a\right)^{2} & =\left(b^{-1} a\right)^{3}, \\
\left(a b^{-1} a\right)^{2} & =\left(b^{2} a^{-1 / 2}\right)^{3}, \\
\left(l r^{-1} l\right)^{-1} s\left(l r^{-1} l\right) & =s^{-1}, \\
s^{-1} r s & =r^{25}, \\
\left(l r^{-1} l\right)^{2} & =\left(s r^{5} l r^{-1} l\right)^{3} .
\end{aligned}
$$

As in the previous two cases, this follows from the presentation of the group $\mathrm{SL}_{2}(\mathbf{Z} / N \mathbf{Z}) \cong \mathrm{SL}_{2}(\mathbf{Z} / e \mathbf{Z}) \times \mathrm{SL}_{2}(\mathbf{Z} / m \mathbf{Z})$ given in [2, Lemma 3.5].

Acknowledgements. This paper is a much-condensed version of the first author's University of Warwick MMath dissertation, written in 2011-12 under the supervision of the second author. We are grateful to Vincent Delecroix for the original observation that Hsu's test should generalize to odd subgroups.

\section{References}

1. H. Behr and J. Mennicke, 'A presentation of the groups PSL(2,p)', Canad. J. Math. 20 (1968) 1432-1438; MR 0236269.

2. T. Hsu, 'Identifying congruence subgroups of the modular group', Proc. Amer. Math. Soc. 124 (1996) 1351-1359; MR 1343700.

3. I. Kiming, M. Schütt and H. A. Verrill, 'Lifts of projective congruence groups', J. Lond. Math. Soc. (2) 83 (2011) 96-120; MR 2763946.

\author{
Thomas Hamilton \\ Premier Pensions Management \\ Corinthian House \\ 17 Lansdowne Road \\ Croydon CRO 2BX \\ United Kingdom
}

\author{
David Loeffler \\ Mathematics Institute \\ University of Warwick \\ Coventry CV4 7AL \\ United Kingdom
}

d.a.loeffler@warwick.ac.uk 